\title{
Evaluation of COX-2, EGFR, and p53 as biomarkers of non- dysplastic oral leukoplakias
}

\author{
Silvia Maria Díaz Prado, José Luis López Cedrún, Ramón Luaces Rey, Vanessa Medina \\ Villaamil, Augusto Álvarez García, Manuel Valladares Ayerbes, e, Luis Antón Aparicio
}

\begin{abstract}
Objective. Identify candidate SEBs (surrogate endpoint biomarkers) for premalignant trends in head and neck mucosa.

Study design. Study, by qPCR (quantitative real-time polymerase chain reaction), the expression of COX-2, EGFR and p53 in 24 biopsies of non-dysplastic oral leukoplakia and contra-lateral normal-appearing mucosa.

Results. COX-2 was up-regulated in leukoplakia (79.2\%); whereas EGFR and p53 were up-regulated $(\mathrm{p}>0.05)$ in oral contra-lateral normal-appearing mucosa (60\% and 46\% respectively). Also, p53 expression was correlated with tobacco smoke habits and Spearman's rank correlation coefficient showed a positive linear correlation between p53 and EGFR mRNA expression levels.

Conclusions

COX-2 would serve as SEB of oral leukoplakia. The results suggest that p53 appears to be one of the molecular targets of tobacco-related carcinogens in leukoplakia and that the co-expression of p53 and EGFR may play a role in this kind of oral pre-cancerous lesion. More detailed studies of EGFR and p53 should be continued in the future.
\end{abstract}

\section{Abbreviations}

SEB, surrogate endpoint biomarker; qPCR, quantitative real-time polymerase chain reaction; HNSCC, head and neck squamous cell carcinoma; SCC, squamous cell carcinoma; PGs, prostaglandins; SCCHN, squamous cell carcinoma of the head and neck; RT-PCR, reverse-transcription polymerase chain reaction; IEN, intraepithelial neoplasia, R.E.L., relative expression level.

Keywords

EGFR; COX-2; p53; Oral leukoplakia; Quantitative real-time PCR; Molecular marker.

\section{Introduction}

Oral premalignant lesions include leukoplakia and erythroplakia, which develop in epithelial surfaces chronically exposed to carcinogens such as tobacco and alcohol. These lesions are markers for field cancerization, because patients with oral premalignancy can develop squamous cell carcinoma at the site of the lesion(s) as well as at other sites in the upper aerodigestive tract. Histologically, oral leukoplakia includes hyperplasia, hyperkeratosis, and dysplasia and carcinoma in situ (Rhee et al., 2004).

The development of an oral squamous cell carcinoma usually involves normal oral mucosa changing to oral leukoplakia or intraepithelial neoplasia (IEN). Oral IEN is an intermediate step between normal epithelium and invasive cancer. This intermediate step includes genetic or epigenetic alterations (eg, alterations of tumor suppressor genes such as FHIT, p16, 53, cyclin D1 overexpression and telomerase activation), loss of cellular control, phenotypic characteristics overlapping those of invasive cancer and a substantial risk of biologically aggressive cancer (Lippman et al., 2005). In a recent study about the behavior of oral premalignant lesions, it has been demonstrated that $7 \%$ harboured a carcinoma, $69 \%$ of lesions showed a degree of epithelial dysplasia or carcinoma in situ, and that $12 \%$ developed carcinoma after a mean follow-up period of 7.5 years (Holmstrup et al., 2007). Leukoplakia is usually located in alveolar, bucal and inferior lip mucous, having a higher percentage of malignancy than lateral lingual and inferior lip. Therefore, anatomic location of leukoplakia may be an important diagnostic indicator.

The ability to intervene prior to the development of a squamous cell carcinoma may improve treatment results (Sudbo and Reith, 2005). This requires the early identification of molecular events that represent early phases of malignant transformation (Sudbo and Reith, 2005). Underlying this histologic transition are multiple molecular and cellular changes, including changes in the expression of growth 
factor/receptor genes. Oral carcinogenesis is a complex multifocal process of multifocal field cancerization (Slaughter et al., 1953) and intraepithelial clonal spread.

Efforts are being made to identify SEBs for head and neck squamous cell carcinoma (HNSCC). Candidate SEBs for invasive squamous cell carcinoma (SCC) of the upper aerodigestive tract are detectable molecular, cellular, and tissue changes that take place during tumorigenesis. The upper aerodigestive tract provides a suitable model for studying the transition, over time, from normal epithelium to the development of squamous cell carcinoma and thus, for the investigation of surrogate endpoint biomarkers (SEBs) of the different stages from early to advanced phases of malignant transformation (Fan et al., 2006). This model's uniqueness derives from multistage tumorigenesis, field cancerization, and ease of access.

Many human malignancies, including HNSCC, produce more prostaglandins (PGs) than the normal tissues from which they arise (Bennett et al., 1980 and Jung et al., 1985). Increased synthesis of PGs in transformed cells and tumors can be a consequence of enhanced expression of COX-2 (Dannenberg et al., 2001). PGs are believed to be important in the pathogenesis of cancer, because of its effects on cell proliferation, angiogenesis, immune surveillance, and apoptosis (Williams et al., 2000, Goodwin \& Geuppens, 1983, Goodwin et al., 1977, Tsujii \& DuBois, 1995 and Pandey et al., 2008). Increased amounts of COX-2 are commonly found in premalignant and malignant conditions, including oral leukoplakia and HNSCC (Dannenberg et al., 2001, Chan et al., 1999, Renkonen et al., 2002, Dannenberg \& Subbaramaiah, 2003 and Dannenberg et al., 2005). There is extensive evidence, beyond the finding that COX-2 is commonly overexpressed in neoplastic tissue, to suggest that COX-2 is mechanistically linked to the development of cancer. Clinical studies have been initiated to determine the chemoprotective effects of selective COX-2 inhibitors in patients with oral leukoplakia and Barrett's esophagus (Altorki et al., 2004).

EGFR, a surface receptor with intrinsic tyrosine kinase activity, is one of several known pivotal intermediates in many epithelial malignancies (Raymond et al., 2000, Shirasuna et al., 1991 and Herbst \& Shin, 2002). The expression of EGFR was up-regulated in oral leukoplakia, in fact its expression levels increased in the stratum spinosum (Srinivasan and Jewell, 2001). In addition, invasive cancer displays a more extreme pattern of overexpression (Shin et al., 1994 and Grandis et al., 1998).

Tumor suppressor genes, like p53, and other proteins associated with the cell-cycle mediate abnormalities in cell proliferation. The overexpression of mutant p53 protein has been studied by a number of authors (Santos Garcia et al., 2005 and López-Martínez et al., 2002), who have detected p53 protein immunopositivity in $27-35 \%$ of premalignant lesions and in 33-100\% of squamous cell carcinoma of the head and neck (SCCHN). The increased p53 expression detected in early-stage SCCHN indicated that $\mathrm{p} 53$ gene activation is implicated in the genesis of these carcinomas. Since p53 disturbances lead to a loss in cell cycle control, a study of p53 gene expression would contribute to an improved diagnosis of oral leukoplakias.

In the present work we decided to study non-dysplastic leukoplakias because there is less knowledge on this kind of lesions than in the dysplastic ones. This may be due, in part, to the fact that this latter lesions rarely end in squamous cell carcinoma and for this reason researchers are not usually interested on them. In this regard in leukoplakia it is generally accepted that histopathological characteristics of no or mild dysplasia has lower risk of malignant transformation than moderate or severe dysplasia.

Most studies based on the investigation of new SEBs in non- or dysplastic oral leukoplakia were performed by immunohistochemistry and not by molecular biology techniques. Only a few work, for example the studies from Pandey et al. (2008) were performed using PCR, but in this paper conventional PCR was used. Immunohistochemical and conventional PCR methodology represent semi-quantitative methods whereas qPCR is more accurate, sensitive, precise, quickly and quantitative with significantly less inter-assay variation. qPCR is also highly reproducible and reliable, characteristics that should be shared by any methodology used for clinical diagnosis.

The objective of this study was to identify candidate SEBs for premalignant trends in head and neck mucosa. In this regard and to better understand the role of COX-2, EGFR and p53 in leukoplakia we examined in vivo the mRNA expression levels of these three markers in 24 samples of oral leukoplakia and contra-lateral normal-appearing mucosa against a pool of healthy human oral mucosa from healthy donors. 


\section{Materials and methods}

\section{Processing of clinical tissue specimens}

This is a case-control paired study in which a total of 24 patients (12 males and 12 females ranging from 42 to 88 years) with non-dysplastic oral leukoplakia were included (Supplementary Table 1). Inclusion criteria: patients up to 18 years with white lesion (leukoplakia) in the oral cavity that after biopsy analysis the absence of inflammatory component and presence of epithelial acanthosis without dysplasia were confirmed.

Exclusion criteria: patients up to 18 years with white lesion (leukoplakia) in the oral cavity that after biopsy analysis the presence of inflammatory component and presence of epithelial acanthosis without dysplasia or absence of inflammatory component and presence of epithelial acanthosis with dysplasia were confirmed. Samples were obtained from the Maxillofacial Surgery Service, A Coruña University Hospital. Clinical characteristics analyzed included age, gender, tobacco status and localization of the non-dysplastic leukoplakia. From each patient, 2 samples were collected: opposed lateral oral mucosa and leukoplakia mucosa. Mucosa and submucosa were dissected from the samples. A hematoxylin-eosin (HE) staining confirmed that the 24 samples analyzed had not an inflammatory component in the submucosa and that the contra-lateral mucosa of all the samples were normal-appearing. A senior pathologist, without prior knowledge of the clinical data, performed the histological study. The pathologist, evaluating the surface epithelium and submucosa of all the samples stained with hematoxylin-eosin, assessed the absence or presence of epithelial acanthosis. Regarding cases with epithelial acanthosis the pathologist also evaluated the absence of dysplastic changes and absence of inflammatory component.

All fresh samples were frozen before RNA extraction. As a control sample, a pool of healthy human oral mucosa from healthy donors $(n=19)$ was used. The H-E staining was also used to confirm that this pool of healthy human oral mucosa from healthy donors was normal. Of the total 19 healthy human oral mucosa from healthy donors, 5 of them were smokers: 1 light smoker (less than 5 cigarettes per day), 2 moderate smokers ( 5 to 15 cigarettes per day) and 2 severe smokers (more than 15 cigarettes per day). Among the 24 patients with oral leukoplakia analyzed, 10 of them were smokers ( 2 light smokers and 8 severe smokers) and 14 of them were non-smokers.

The study was approved by the Institutional Review Board of the Ethics Committee of Clinical Investigation in Galicia (Spain). All samples were confirmed by histological analysis. Tissue samples were dissected under stringent sterile conditions to prevent RNA contamination and were immediately frozen in liquid nitrogen and stored at $-80^{\circ} \mathrm{C}$ until the time of RNA extraction.

\section{RNA extraction}

Total RNA isolation from frozen healthy and leukoplakia specimens was performed, following the manufacturer's protocols, using Tripure Isolation Reagent (Roche, Mannheim, Germany). For the isolation of each tissue sample, 50 to $100 \mathrm{mg}$ was processed. Total RNA was treated with DNase I.

RNA integrity was confirmed by $2 \%$ agarose gel electrophoresis stained with ethidium bromide. RNA was also assessed for quantity at $260 \mathrm{~nm}$ using a Genious UV spectrophotometer. $A_{260} / A_{280}$ relation was calculated for quality, quantity and purity.

Standard procedures for manipulation of nucleic acids were essentially those previously reported (Sambrook et al., 1989).

\section{cDNA synthesis}

Reverse-transcription PCR (RT-PCR) was performed using Super-Script ${ }^{\text {TM }}$ First-Strand Synthesis System for RT-PCR (Invitrogen, Spain) up to a total volume of $20 \mu \mathrm{l}$. One microgram of total RNA, $2.5 \mathrm{nM}$ random hexamers, $0.5 \mathrm{mM}$ dNTP mix, and $3 \mu \mathrm{l}$ of DEPC-treated water were denatured at $65^{\circ} \mathrm{C}$ for $5 \mathrm{~min}$ and chilled on ice for at least $1 \mathrm{~min}$. Also, $2 \mu \mathrm{l}$ of $10 \times \mathrm{RT}$ buffer, $5 \mathrm{mM} \mathrm{MgCl}, 0.01 \mathrm{M} \mathrm{DTT}$, and $40 \mathrm{U}$ of RNase-OUT ${ }^{\mathrm{TM}}$ Recombinant Ribonuclease Inhibitor were mixed, collected by centrifugation, and incubated at $25^{\circ} \mathrm{C}$ for $2 \mathrm{~min}$. After incubation, $50 \mathrm{U}$ of Super-Script ${ }^{\mathrm{TM}}$ II RT was added and incubated at $25^{\circ} \mathrm{C}$ for $10 \mathrm{~min}, 42^{\circ} \mathrm{C}$ for $50 \mathrm{~min}$, and $70^{\circ} \mathrm{C}$ for $15 \mathrm{~min}$ in a Thermocycler (GeneAmp® PCR System 9700, Applied Biosystems, Spain). Finally, samples were chilled on ice and incubated with 2 U of RNAse $\mathrm{H}$ for $20 \mathrm{~min}$ at $37^{\circ} \mathrm{C}$ before proceeding to amplification of the target cDNA. Positive and negative controls were included in each experiment.

RNA extraction, reverse-transcription PCR assay setup and post reverse-transcription PCR product analysis were carried out in separate designated rooms to prevent cross-contamination. cDNA was 
quantified and assessed for purity using a Genious UV spectrophotometer. cDNA concentration was measured at $260 \mathrm{~nm}$. Also $A_{260} / A_{280}$ relation was calculated in order to find cDNA quality, quantity and purity.

\section{Quantitative real-time PCR $(q P C R)$}

Quantitative real-time PCR analysis was performed, using LightCycler® 480 SYBR Green I Master Kit (Roche), on a LightCycler® 480 Real Time PCR System (Roche). PCR reaction consisted of $10 \mu$ of Master Mix $2 \times$ conc., $0.35 \mu \mathrm{M}$ of each forward and reverse primer, template cDNA and PCR-grade water up to a final volume of $20 \mu \mathrm{l}$ in the LightCycler ${ }^{\circledR} 480$ Multiwell Plate 96. The Multiwell plate was centrifuged at $3000 \mathrm{rpm}$ for $2 \mathrm{~min}$ and was loaded in the LightCycler ${ }^{\circledR} 480$ Instrument until the PCR program started. An initial activation at $95^{\circ} \mathrm{C}$ for $5 \mathrm{~min}$ followed by an amplification target sequence 50 cycles of $95^{\circ} \mathrm{C}$ for $10 \mathrm{~s}, 59-60^{\circ} \mathrm{C}$ (depending on the primer pair used) for $10 \mathrm{~s}$, and $72^{\circ} \mathrm{C} \mathrm{9-20} \mathrm{s}$ (depending on the amplified amplicon size) was used. For melting curve analysis, 1 cycle of $95^{\circ} \mathrm{C}$ for $5 \mathrm{~s}$, $70^{\circ} \mathrm{C}$ for $15 \mathrm{~s}$, and $95^{\circ} \mathrm{C}$ for $1 \mathrm{~s}$ was used. Finally, a cooling step was used at $40^{\circ} \mathrm{C}$ for $10 \mathrm{~s}$. PCR primers for the amplification of the different COX-2, EGFR and p53 genes were carefully designated using the web-based ProbeFinder software (Universal ProbeLibrary Design Center) accessible at Roche Applied Science, 2005 or via Roche Applied Science home page. PCR primers were positioned to span exon-intron boundaries in order to reduce the risk of detecting genomic DNA. Primers were purchased from Invitrogen (Spain). The following list summarizes the primer pairs used: COX-2: 5' CCT TCG AAA TGC AAT TAT GAG TT 3' (forward); 5' CAC AGG AGG AAG GGC TCT AGT 3' (reverse). EGFR: 5' CAG CCA CCC ATA TGT ACC ATC 3' (forward); 5' AAC TTT GGG CGA CTA TCT GC 3' (reverse). p53: 5' AGG CCT TGG AAC TCA AGG AT 3' (forward); 5' CCC TTT TTG GAC TTC AGG TG 3' (reverse). HPRT: 5' TGA CCT TGA TTT ATT TTG CAT ACC 3' (forward); 5' CGA GCA AGA CGT TCA GTC CT 3' (reverse). Suitable selection of housekeeping gene was performed using Human Endogenous Control Gene Panel (TATAA Biocenter). The Excel macro named GeNorm VBA applet for Microsoft Excel was used to determine the gene with most correlated expression in the set of samples. HPRT (hypoxanthine-guanine phosphoribosyltransferase) was selected as the most stable housekeeping gene. Also, this internal control was used to verify integrity of RNA and efficacy of reverse-transcription. Any specimen with inadequate housekeeping mRNA level was excluded from the study.

The expected size of each qPCR product was confirmed by $1.8 \%$ agarose gel electrophoresis. Furthermore, at least one qPCR product of each qPCR experiment was analyzed by DNA sequencing. All qPCR products revealed a single band corresponding to the single-amplified products as should predict the melting curve analysis of the qPCR.

Data analysis was performed with LightCycler® 480 Relative Quantification software (Roche). Relative levels of expression were calculated by the $2^{-\Delta \Delta C t}$ method (Livak and Schmittgen, 2001). Each assay was done at least in triplicate and included marker-positive and marker-negative controls and reagent with no template controls.

\section{DNA sequencing}

COX-2, EGFR and p53 exons were sequenced because in qPCR experiments we used the SYBR Green I fluorescent dye as a non-specific method to detect the fluorescence. In order to verify the specific amplification of the qPCR product we confirmed the sequence of each amplicon by sequencing them. For this purpose at least one qPCR product from each quantitative real-time PCR experiment was used as template DNA. Products were purified by enzymatic method (ExoSAP-IT, Amersham USB). DNA sequencing was performed on an ABI 3700 system (Applied Biosystems). Specific primers for DNA sequencing were the same as for the quantitative real-time PCR experiments.

\section{Immunohistochemistry}

To investigate the presence of COX-2, EGFR and p53 proteins we used Chemmate ${ }^{\mathrm{TM}}$ DAKO EnVision ${ }^{\mathrm{TM}}$ Detection Kit, Peroxidase/DAB, Rabbit/Mouse (provided by Dako, Carpinteria, CA, USA) the following manufacturer's instructions. This kit uses peroxidase and diaminobenzidine (DAB) for developing. Briefly, after de-paraffining with xylene, gradually rehydratting, antigenic retrieval and blocking endogenous peroxidase by pre-treatment with citrate buffer in a pressure cooker, a monoclonal antibody specific to human COX-2 (Abcam Ltd., Cambridge, UK), EGFR (Abcam) and p53 (Abcam) was added and incubated overnight. Then, sections were incubated with secondary antibody. Substrate chromogen (DAB) was added. Afterward, slides were mounted with DePex Mounting Medium (BDH Chemicals, 
Ltd.). Also at least three slides were processed without primary antibody. Negative staining controls omitted the primary monoclonal antibody. Positive staining controls were those indicated on the datasheet of each antibody. Samples were examined using an optical microscope. For the evaluation of the immunohistochemical staining an investigator, without prior knowledge of the clinical follow-up data, assessed COX-2, EGFR and p53 protein expression.

\section{Statistical analysis of the results}

Statistical analysis of the results was realized with SPSS program (version 14.0). The comparison of medians of each marker analyzed (COX-2, EGFR and p53) in contra-lateral normal-appearing and leukoplakia mucosa was performed using non-parametric statistics, signed rank test. The relationship between COX-2, EGFR and p53 expression values and clinicoanatomo-pathological data were also studied by non-parametric statistics, Mann-Whitney $U$ and Kruskal-Wallis tests. Statistical significance of differences was evaluated at 95\% confidence level and $p$ values $<0.05$ were considered to be significant. Also non-parametric correlations were measured by Spearman's rank correlation coefficient.

\section{Results}

\section{Expression of COX-2, EGFR and p53 in oral leukoplakias}

To assay COX-2, EGFR and p53 gene expression as potential markers associated with oral leukoplakias, we analyzed 24 samples of oral leukoplakia and contra-lateral normal-appearing mucosa against a pool of healthy human oral mucosa from healthy donors, by means of qPCR (Fig. 1 and Fig. 2). As expected the mRNA expression studies among the patients showed the existence of donor-dependent variance.
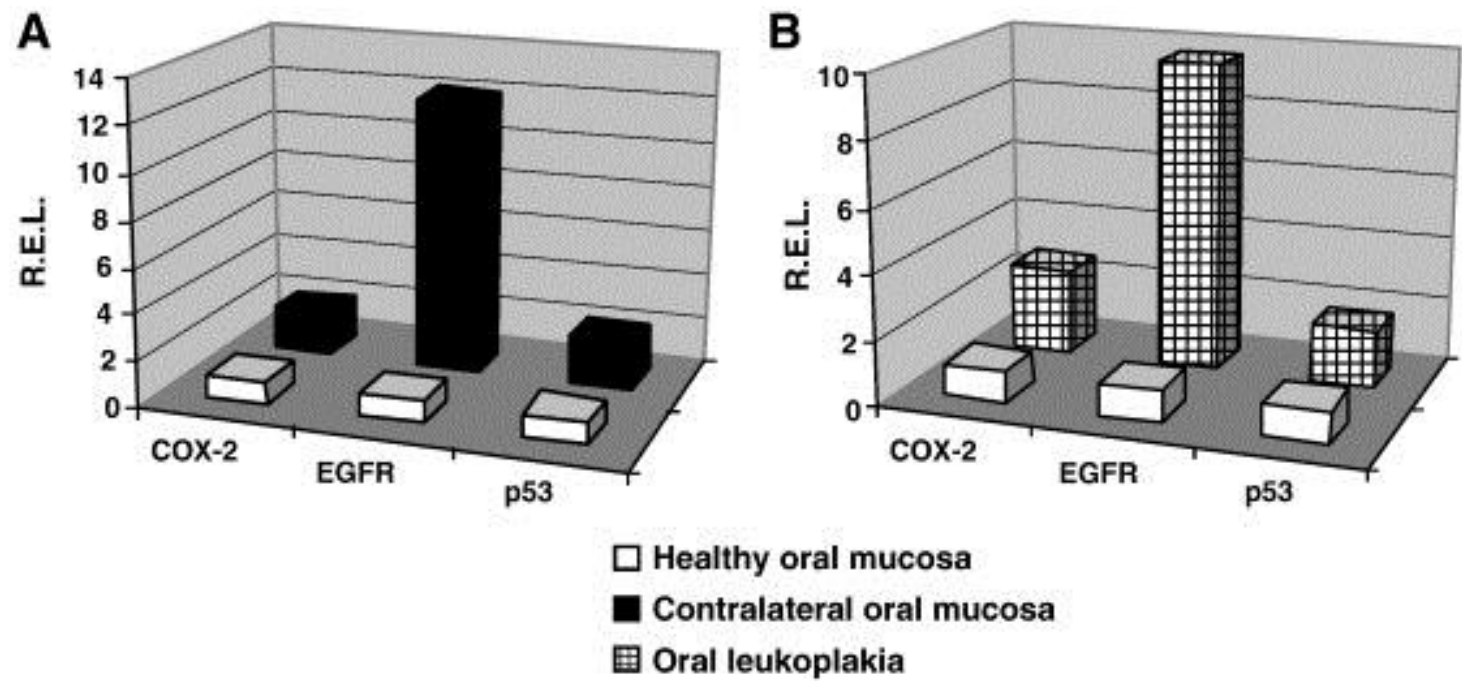

Fig. 1. COX-2, EGFR and p53 mRNA expression levels in contra-lateral normal-appearing mucosa $(n=24)(\mathrm{A})$ and in oral leukoplakia $(n=24)(\mathrm{B})$ against a pool of healthy human oral epithelium $(n=19)$. $X$-axis represents the different human genes tested, $Y$-axis represents the different tissue samples where the expression levels were studied and $Z$-axis represents COX-2, EGFR and p53 mRNA relative expression levels (R.E.L.) respectively. mRNA levels were measured by quantitative real time PCR as described in " Materials and methods". qPCR results are the mean of at least three independent measurements. Data were normalized against the values obtained in healthy human oral mucosa, which were considered equal to 1 . 


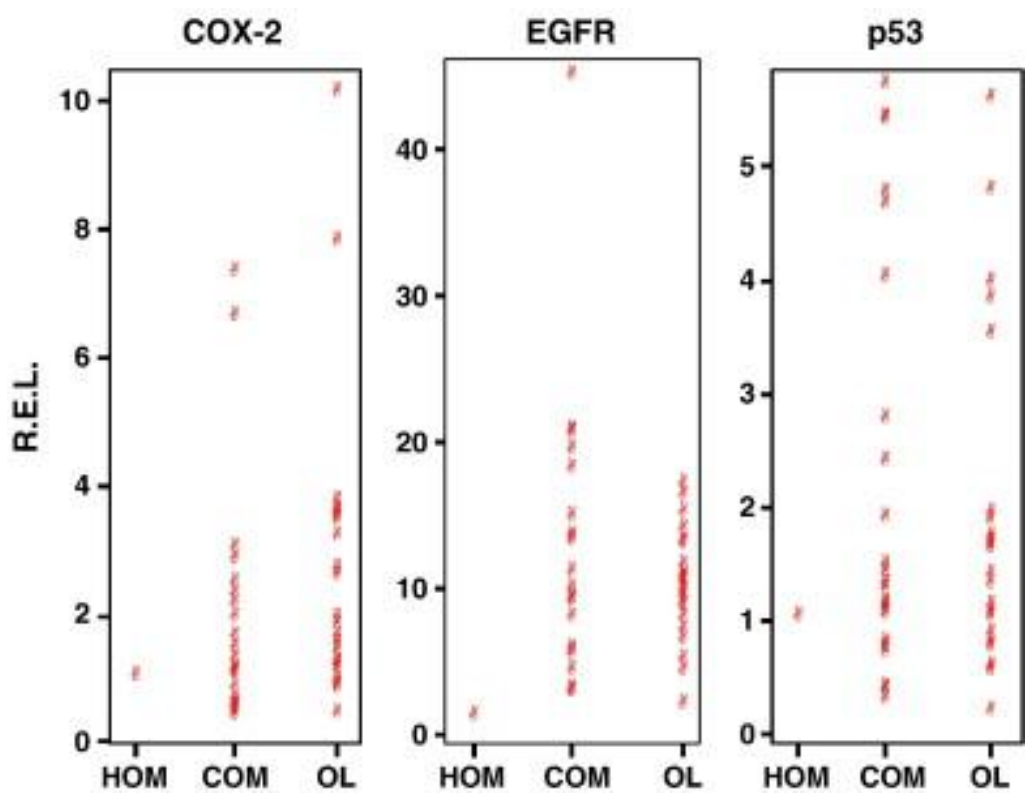

Fig. 2. mRNA expression levels of COX-2, EGFR and p53 ( $Y$-axis) determined in healthy oral mucosa from healthy donors (HOM) and contra-lateral normal-appearing mucosa $(\mathrm{COM})$ and oral leukoplakia $(\mathrm{OL})$ from patients $(X$-axis). mRNA levels were measured by qRT-PCR as described in "Materials and methods". In the graph, each point represents the mean of at least three independent measurements of each sample specimen analyzed.

The expression levels of COX-2 were higher in oral leukoplakia compared with contra-lateral normalappearing mucosa samples, which showed lower values (means: 2.66 versus 1.87 respectively, signed rank test, $p=0.019)$. In this way, $79.2 \%$ of the samples analyzed showed higher COX-2 expression levels in oral leukoplakia than in opposed lateral oral mucosa. COX-2 was down-regulated in the pool of healthy human oral mucosa from healthy donors in such a way that in this group of samples the lowest expression values of COX-2 were obtained ( Table 1 ).

Table 1. Comparison of mRNA R.E.L. of the 3 markers assessed among the different tissue samples analyzed.

\begin{tabular}{|c|c|c|c|}
\hline $\begin{array}{l}\text { Gene } \\
\text { assessed }\end{array}$ & Comparison among tissue sample analyzed & & $\begin{array}{l}U \text { Mann-Whitney } \\
\text { test }\end{array}$ \\
\hline \multirow[t]{2}{*}{$\mathrm{COX}-2$} & $\begin{array}{l}\text { Healthy human oral mucosa from healthy } \\
\text { donors }\end{array}$ & $\begin{array}{l}\text { Contra-lateral normal-appearing } \\
\text { mucosa }\end{array}$ & $p=0.005$ \\
\hline & & Oral leukoplakia & $p=0.000$ \\
\hline \multirow[t]{2}{*}{ EGFR } & $\begin{array}{l}\text { Healthy human oral mucosa from healthy } \\
\text { donors }\end{array}$ & $\begin{array}{l}\text { Contra-lateral normal-appearing } \\
\text { mucosa }\end{array}$ & $p=0.00$ \\
\hline & & Oral leukoplakia & $p=0.00$ \\
\hline \multirow[t]{2}{*}{ p53 } & $\begin{array}{l}\text { Healthy human oral mucosa from healthy } \\
\text { donors }\end{array}$ & $\begin{array}{l}\text { Contra-lateral normal-appearing } \\
\text { mucosa }\end{array}$ & $p=0.005$ \\
\hline & & Oral leukoplakia & $p=0.005$ \\
\hline
\end{tabular}

Regarding the EGFR gene, the expression values obtained in oral leukoplakia were lower than in normal-appearing mucosa, although this difference was not statistically significant (means: 9.66 versus 12.07 respectively, signed rank test, $p>0.05$ ). In this way, $60 \%$ of the samples analyzed showed a higher expression in contra-lateral normal oral epithelium than in oral leukoplakia. EGFR expression values were lower in the pool of healthy human oral mucosa from healthy donors than in the oral leukoplakia and contra-lateral normal-appearing mucosa of patient samples ( Table 1).

As for the p53 gene, expression levels were also higher in normal-appearing mucosa, although, as with the EGFR gene, this difference was statistically insignificant (means: 2.1 versus 1.83 respectively, 
signed rank test, $p>0.05)$. Therefore, when comparing opposed lateral oral mucosa and oral leukoplakia from the same patient, $54.2 \%$ of samples showed higher p53 expression in opposed lateral oral mucosa than in oral leukoplakia. p53 expression was also down-regulated in the pool of healthy human oral mucosa from healthy donors compared with that in oral leukoplakia and contra-lateral normal-appearing mucosa of patient samples ( Table 1).

Immunohistochemical stainings were carried out to identify the epithelial cells which express the three biomarkers studied in all the contra-lateral normal and leukoplakia mucosa samples (Supplementary

Fig. 1). In this regard, p53 showed positivity in basal cells from the basal epithelium and EGFR showed positivity in cells of the lower third of the basal epithelium. For COX-2 and in some cases, the assessment was more complicated because it is a cytoplasmic marker. Basal and parabasal layers showed expression of COX-2.

\section{Correlation of COX-2, EGFR and p53 gene expression with clinicopathological parameters}

mRNA expression levels of COX-2, EGFR and p53 genes tested in oral leukoplakias were correlated, by means of non-parametric statistics, with different clinicopathological parameters that included sex, age, tobacco smoke habits, and leukoplakia site (Supplementary Table 2). Patients' survival was not analyzed because all the leukoplakia patients were alive at the time of going to press. During follow-up time longer than 1 year, 1 patient showed no improvement, 6 patients showed partial improvement, 8 patients showed great improvement, 5 patients showed almost healing and 4 patients showed healing. Only 2 of the 24 patients analyzed developed further lesions: 1 of them developed tongue carcinoma and the other 1 developed lung carcinoma. In addition, among all genes tested, p53 expression levels were correlated with tobacco smoke habits (Mann-Whitney $U$ test, $p=0.043$ ), in such a way that higher p53 expression levels were observed for tobacco smoke patients than for non-smokers (means: 2.378 versus 1.347 respectively). There were no correlations with sex, age and localization of the leukoplakia. However, by means of non-parametric statistics (Spearman's rank correlation coefficient), we tested that there is a positive linear correlation between EGFR and p53 mRNA expression levels (Spearman rho $=0.6, n=24$, $p=0.003$ ) ( Supplementary Fig. 2).

\section{Discussion}

Oral cancer is a major health problem in many parts of the world and is associated with significant morbidity and mortality. Malignancy of the oral cavity is often preceded by premalignant lesions, of which leukoplakia is the most common premalignant or potentially malignant lesion of the oral mucosa, and therefore numerous epidemiologic studies have been carried out worldwide regarding the prevalence of this alteration (Santhi et al., 2006 and Kövesi \& Szende, 2003). The annual percentage of malignant transformation of leukoplakia varies in different parts of the world, owing to variations in the use of tobacco and dietary habits. The rate of malignant transformation varies from practically $0 \%$ to almost $20 \%$ in 1 to 30 years (Santhi et al., 2006). The molecular mechanisms leading to and involved in the malignant transformation of normal oral tissue and leukoplakia are still poorly understood. Characterization of the molecular progression of a particular tumor type is critical in providing markers for diagnostic molecular assays.

COX-2, an inducible isoform of cyclooxygenase (Aparicio et al., 2007), is undetectable (or detectable at low levels) in most normal tissues, but is rapidly induced in response to inflammatory or mitogenic stimuli, including cytokines, growth factors, and tumor promoters (Pandey et al., 2008, Dannenberg et al., 2005 and Choe et al., 2005). Multiple mechanisms are likely to account for overexpression of COX-2. The latter is normally regulated at both transcriptional and post-transcriptional levels, and can also be regulated by the rate of protein synthesis and/or degradation. Multiple lines of evidence show that COX-2 has a significant role in carcinogenesis and is overexpressed in transformed cells, premalignant and malignant tissues, including those of the head and neck (Chan et al., 1999, Dannenberg et al., 2005, Santhi et al., 2006 and Choe et al., 2005). Expression of COX-2 is present in various neoplasms, such as colorectal, lung, stomach, pancreas, breast, urinary, bladder and esophageal neoplasms (Pandey et al., 2008). Selective COX-2 inhibitors suppress the growth of cells by inducing G0/G1, cell-cycle arrest and, therefore, apoptosis (Pandey et al., 2008). In the present study we observed that in oral leukoplakia from patients COX-2 gene expression was up-regulated with regard to contra-lateral normal-appearing mucosa samples. The statistical significance found among oral leukoplakia and contra-lateral normal-appearing mucosa tissues suggests that COX-2, besides being an inflammation biomarker induced by the nonconstitutive form of the cyclooxygenase, could represent a SEB of oral leukoplakia. 
In agreement with our COX-2 mRNA expression results, previous reports showed increased COX-2 mRNA or protein expression in pre-cancerous lesions and oral cancer (Pandey et al., 2008, Akita et al., 2004, Banerjee et al., 2002, Chan et al., 1999, Mestre et al., 1999, Mohan \& Epstein, 2003, Moraitis et al., 2005, Nishimura et al., 2000, Renkonen et al., 2002, Sakamoto et al., 2004, Taketo, 1998, Terakado et al., 2004, Tsai et al., 2004 and Ye et al., 2004) . It is well known that COX-2 overexpression contributes to many aspects of carcinogenesis, such as inhibition of apoptosis, promotion of cell proliferation, induction of angiogenesis, and increasing invasiveness (Choe et al., 2005, Turini \& Dubois, 2002 and Cao \& Prescott, 2002). In particular, the potential contributions of COX-2 overexpression to tumorigenesis are related with the increased production of mutagens: malondialdehyde (MDA) can be produced by isomerization of $\mathrm{PGH}_{2}$ both enzymatically and non-enzymatically. Additional carcinogens can be formed by oxidation of aromatic amines, heterocyclin amines, and dihydrodiol derivatives of polycyclic hydrocarbons (Wiese et al., 2001). Thus, COX-2 overexpression may lead to DNA damage, thereby contributing to carcinogenesis. In another respect, much interest has focused on the ability of COX-2 to suppress apoptosis. Diminished apoptosis is thought to favour carcinogenesis by permitting the survival of cells that have acquired mutations, and thus is viewed as one of the central mechanisms of tumorigenesis (Sheng et al., 1998 and Chan et al., 1998). On the other hand, it has become apparent that cyclooxygenases are involved in angiogenesis (Cao et al., 2000). COX-2 apparently contributes to the production of proangiogenic factors, including vascular endothelial growth factor (VEGF), transforming growth factor-1 (TGF-1), platelet-derived growth factor (PDGF), and endothelin-1. Additionally, COX-2 is responsive to several oncogenes, including v-src, v-Ha-ras, HER-2/neu, and Wnt genes (Xie \& Herchman, 1995, Subbaranmaiah et al., 1999, Howe et al., 1999 and Vadlamudi et al., 1999). Recently it has been proposed that the Wnt signaling pathway interacts among cyclooxygenase- 2 and Ku expression. Interactions between Wnt signaling and $\mathrm{Ku}$ factor were indirectly regulated by COX-2 (Chang et al., 2008). $\mathrm{Ku}$ is an important factor influencing the DNA repair process (Chang et al., 2008).

p53 is a well known tumor suppressor gene located on the short arm of chromosome 17, and mutation in this gene occurs in $43 \%$ of HNSC patients (Rhee et al., 2004). It possesses multiple functions, since it appears to be implicated not only in control of the cell cycle but also in DNA integrity and in the survival of cells exposed to DNA-damaging agents (López-Martínez et al., 2002). Expression of p53 is strictly regulated in normal cells. The overexpression of p53 protein is the most common genetic alteration in head and neck cancer (Fan et al., 2006, Duarte et al., 2008 and Kurokawa et al., 2003). Earlier immunohistochemical studies showed that patients with p53 expression have a higher risk of developing tongue cancer than those who do not show p53 expression (Vora et al., 2006). There is a large amount of valuable data confirming that p53 immunohistochemical staining was a clinically useful supplement of histopathological assessment in the prognosis of oral leukoplakia. In this way, it has been well documented in previous reports that deregulation of p53 is a valuable biomarker to predict malignant transformation in premalignant oral lesions; since it seems that its overexpression increases with the degree of histopathological severity of these lesions (Fan et al., 2006 and Santos Garcia et al., 2005). In our study, we observed that p53 expression values showed a slight decrease in oral leukoplakia with regard to contra-lateral normal-appearing mucosa but that this was not statistically significant $(p>0.05)$. However, Fan et al. (2006) detected a statistically more significant decrease in p53 protein expression in leukoplakia than that in normal mucosa.

Although, in the present study, p53 expression was higher in oral leukoplakia than in the pool of healthy human oral mucosa from healthy donors, which is in accordance with the literature, since normal epithelium practically does not express this protein (Lawall and Crivelini, 2006). p53 appears to be one of the molecular targets of tobacco-related carcinogens in oral cancer (Vora et al., 2006). In this way, we also demonstrated the existence of a correlation between p53 mRNA expression levels and tobacco smoke habit, since higher p53 expression levels were observed for tobacco smoke patients. This could be of great importance in oral premalignant lesions, since most of the oral cancer patients are tobacco smokers. At present, it is well known that tobacco may cause oral cancer, mainly via effects on p53, since altered p53 expression is associated with increased genomic instability in oral intraepithelial neoplasia (IEN), and may cause acceleration in the rate of genetic alterations during oral tumorigenesis (Lippman et al., 2005).

EGFR is a member of the ErbB surface receptor tyrosine kinase family and is one of several known pivotal intermediates in many epithelial malignancies (Choe et al., 2005, Raymond et al., 2000 and Lippman et al., 2005). EGFR overexpression occurs between $80 \%$ and $100 \%$ of premalignant and malignant lesions of the oral cavity (Lippman et al., 2005). In addition, its expression has been observed in several malignancies including head and neck, lung, breast, prostate, bladder and pancreatic cancers (Rhee et al., 2004). Although earlier studies considered that EGFR and COX-2 signaling pathways contributed to carcinogenesis, angiogenesis and chemoresistance (Ali et al., 2005), more recent studies show evidence that indicates the existence of tight crosstalk between these two pathways 
(Dannenberg et al., 2005, Choe et al., 2005, Lippman et al., 2005 and Ali et al., 2005). Activation of EGFR induces COX-2, mainly via the mitogen-activated protein kinase pathway. On the other hand, prostaglandins, produced by COX-2, can induce EGFR activation (Choe et al., 2005). Both EGFR and COX-2 affect the same aspects of carcinogenesis, such as inhibition of apoptosis and induction of angiogenesis (Choe et al., 2005). Moreover, the interaction between EGFR and COX-2 can potentially increase the carcinogenic process (Dannenberg et al., 2005). In the present study we observed that EGFR expression values showed a slight decrease in oral leukoplakia, i.e. EGFR was up-regulated in contralateral normal-appearing mucosa samples with regard to oral leukoplakia and the pool of healthy human oral mucosa from healthy donors. These results are in accordance with previous studies, in which it was demonstrated that expression of EGFR and its ligands, transforming growth factor- $\alpha$ or epidermal growth factor, are up-regulated in histologically normal epithelium adjacent to invasive cancer, compared with control normal epithelium in individuals without cancer (Herbst \& Shin, 2002 and Choe et al., 2005). At physiological levels, the expression values of EGFR in normal mucosa should always be positive, since EGFR is the target of the EGF secreted by the salivary glands as mitogen in mucosa proliferation. Mucosa is renewed by means of the EGF that is liberated. The high expression levels of EGFR found in contra-lateral normal-appearing mucosa of patients with leukoplakia with regard to the pool of healthy human oral epithelium from healthy donors (more than 12-fold) could indicate that a 'field cancerization' has been induced (Rhee et al., 2004, Slaughter et al., 1953 and Lippman et al., 2005).

Over fifty years ago the term 'field cancerization' was coined to describe the histologic abnormalities detected in the grossly normal epithelium adjacent to resected SCC. Slaughter et al. (1953) observed a $10 \%$ incidence of subsequent second primaries in their patient population and concluded that this neoplastic multicentricity arose from a preconditioned field of epithelium exposed to carcinogens that were breaking down into cancer at multiple points. More recently, the discovery of genetic alterations supports this theory (Rhee et al., 2004). These events comprise the multi-step process, which includes inherited genetic alterations, damage from carcinogens such as tobacco, alcohol and viral infections (Rhee et al., 2004). Furthermore, our data demonstrated that p53 mRNA expression was positively correlated with EGFR expression in oral leukoplakia. These results suggest that the co-expression of p53and EGFR may play a role in oral pre-cancerous lesions, and also that derangements in p53 function may impact malignant transformation through the direct or indirect effects caused by EGFR overexpression.

\section{Conclusion}

It can be concluded that $\mathrm{COX}-2$ is up-regulated in pre-cancerous lesions. COX-2, besides being an inflammation biomarker induced by the non-constitutive form of the cyclooxygenase, would serve as SEB of oral leukoplakia. The results suggest that p53 appears to be one of the molecular targets of tobacco-related carcinogens in leukoplakia and that the co-expression of p53 and EGFR may play a role in this kind of oral pre-cancerous lesion. Studies into EGFR and p53 should be continued in more detail.

\section{Conflicts of interest}

The authors declare that they have no competing interests.

\section{Authors' contributions}

Conception and design: S.M. Díaz Prado, L.M. Antón Aparicio.

Provision of study materials and patients: J.L. López Cedrún, R. Luaces Rey.

Collection and assembly of data: S.M. Díaz Prado, V. Medina Villaamil.

Data analysis and interpretation: S.M. Díaz Prado, A. Álvarez García, L.M. Antón Aparicio.

Manuscript writing: S.M. Díaz Prado, M. Valladares Ayerbes, L.M. Antón Aparicio.

Final approval of manuscript: S.M. Díaz Prado, J.L. López Cedrún, R. Luaces Rey, A. Álvarez García, M. Valladares Ayerbes, V. Medina Villaamil, L.M. Antón Aparicio.

\section{Acknowledgments}

The authors wish to thank Mrs. M. Haz Conde and I. Santamarina Caínzos for their technical assistance. S.M. Díaz Prado is supported by an Isidro Parga Pondal research contract by the Xunta de Galicia (A Coruña, Galicia, Spain). Cancer research in our laboratory is supported by the "Fundación Juan CanalejoMarítimo de Oza”. 


\section{References}

Akita et al., 2004. Y. Akita, K. Kozaki, A. Nakagawa, et al. Cyclooxygenase-2 is a possible target of treatment approach in conjunction with photodynamic therapy for various disorders in skin and oral cavity. Br. $\mathrm{J}$. Dermatol., 151 (2004), pp. 472-480.

Ali et al., 2005. S. Ali, B.F. El-Rayes, F.H. Sarkar, et al. Simultaneous targeting of the epidermal growth factor receptor and cyclooxygenase-2 pathways for pancreatic cancer therapy. Mol. Cancer Ther., 4 (2005), pp. 19431951.

Altorki et al., 2004. N.K. Altorki, K. Subbaramaiah, A.J. Dannenberg. COX-2 inhibition in upper aerodigestive tract tumors. Semin. Oncol., 31 (2 Suppl 7) (2004), pp. 30-36.

Aparicio et al., 2007. G. Aparicio, D. Prado, P. Jiménez Fonseca, et al. Cyclooxygenase-2 (COX-2): a molecular target in prostate cancer. Clin. Transl. Oncol., 9 (2007), pp. 694-702.

Banerjee et al., 2002. A.G. Banerjee, V.K. Gopalakrishnan, I. Bhattacharya, et al. Deregulated cyclooxygenase-2 expression in oral premalignant tissues. Mol. Cancer Ther., 1 (2002), pp. 1265-1271.

Bennett et al., 1980. A. Bennett, R.L. Carter, I.F. Stamford, et al. Prostaglandin/like material extracted from squamous cell carcinomas of the head and neck. Br. J. Cancer, 41 (1980), pp. 204-208.

Cao et al., 2000. T. Cao, A.T. Pearman, G.A. Zimmerman, et al. Intracellular unesterified arachidonic acid signals apoptosis. Proc. Natl Acad. Sci., 97 (2000), pp. 11280-11285.

Cao \& Prescott, 2002. Y. Cao, S.M. Prescott. Many actions of cyclooxygenase-2 in cell dynamics and in cancer. J. Cell. Physiol., 190 (2002), pp. 279-286.

Chan et al., 1999. G. Chan, J.O. Boyle, E.K. Yang, et al. Cyclooxygenase-2 expression is upregulated in squamous cell carcinoma of the head and neck. Cancer Res., 59 (1999), pp. 991-994.

Chan et al., 1998. T.A. Chan, P.J. Morin, B. Vogelstein, et al. Mechanisms underlying non-steroidal antiinflammatory drug-mediated apoptosis. PNAS, 95 (1998), pp. 681-686.

Chang et al., 2008. H.W. Chang, J.L. Roh, E.J. Jeong, et al. Wnt signaling controls radiosensitivity via cyclooxygenases-2-mediated Ku expression in head and neck cancer. Int. J. Cancer, 122 (2008), pp. 100-107

Choe et al., 2005. M.S. Choe, X. Zhang, H.J.C. Shin, et al. Interaction between epidermal growth factor receptor and cyclooxygenase 2 mediated pathways and its implications for the chemoprevention of head and neck cancer. Mol. Cancer Ther., 4 (2005), pp. 1448-1455.

Dannenberg et al., 2001. A.J. Dannenberg, N.K. Altorki, J.O. Boyle, et al. Inhibition of cyclooxygenase-2: an approach to preventing cancer of the upper aerodigestive tract. Ann. NY Acad. Sci., 952 (2001), pp. 109-115.

Dannenberg et al., 2005. A.J. Dannenberg, S.M. Lippman, J.R. Mann, et al. Cyclooxygenases-2 and epidermal growth factor receptor: pharmacologic targets for chemoprevention. J. Clin. Oncol., 23 (2005), pp. 254-266.

Dannenberg \& Subbaramaiah, 2003. A.J. Dannenberg, K. Subbaramaiah. Targeting cyclooxygenase-2 in human neoplasia: rationale and promise. Cancer Cell, 4 (2003), pp. 431-436.

Duarte et al., 2008. E.C. Duarte, D.C. Ribeiro, M.V. Gomez, et al. Genetic polymorphisms of carcinogen metabolizing enzymes are associated with oral leukoplakia development and p53 overexpression. Anticancer Res., 28 (2008), pp. 1101-1106.

Fan et al., 2006. G.-K. Fan, J. Chen, F. Ping, et al.. Immunohistochemical analysis of P57(kip2), p53 and hsp60 expressions in premalignant and malignant oral tissues. Oral Oncol., 42 (2006), pp. 147-153.

GeNorm, 2006. GeNorm VBA applet for Microsoft Excel [Excel macro on Internet] [cited July 2006]. Available from http://medgen.ugent.be/-jvdescomp/genorm/

Goodwin et al., 1977. J.S. Goodwin, A.D. Bankhurst, R.P. Messner. Suppression of human T-cell mitogenesis by prostaglandin: existence of a prostaglandin producing suppressor cell. J. Exp. Med., 146 (1977), pp. 1719-1731.

Goodwin \& Geuppens, 1983. J.S. Goodwin, J.L. Geuppens. Regulation of immune response by prostaglandins. J. Clin. Immunol., 3 (1983), pp. 295-315.

Grandis et al., 1998. J.R. Grandis, D.J. Tweardy, M.F. Melhem. Asynchronous modulation of transforming growth factor $\alpha$ and epidermal growth factor receptor protein expression in progression of premalignant lesions to head and neck squamous cell carcinoma. Clin. Cancer Res., 4 (1998), pp. 13-20.

Herbst \& Shin, 2002. R.S. Herbst, D.M. Shin. Monoclonal antibodies to target epidermal growth factor receptorpositive tumors: a new paradigm for cancer therapy. Cancer, 94 (2002), pp. 1593-1611.

Holmstrup et al., 2007. P. Holmstrup, P. Vedtofte, J. Reibel, et al. Oral premalignant lesions: is a biopsy reliable?. J. Oral Pathol. Med., 36 (2007), pp. 262-266.

Howe et al., 1999. L.R. Howe, K. Subbaramaiah, W.J. Chung, et al. Transcriptional activation of cyclooxygenase-2 in Wnt-1 transformed mouse mammary epithelial cells. Cancer Res., 59 (1999), pp. 1572-1577.

Jung et al., 1985. T.K. Jung, N.T. Berlinger, S.K. Juhn. Prostaglandins in squamous cell carcinoma of the head and neck: a preliminary study. Laryngoscope, 95 (1985), pp. 307-312.

Kövesi \& Szende, 2003. G. Kövesi, B. Szende. Changes in apoptosis and mitotic index, p53 and ki67 expression in various types of oral leukoplakia. Oncology, 65 (2003), pp. 331-336.

Kurokawa et al., 2003. H. Kurokawa, S. Matsumoto, T. Murata, et al. Immunohistochemical study of syndecan-1 downregulation and the expression of p53 protein or ki-67 antigen in oral leukoplakia with or without epithelial dysplasia. J. Oral Pathol. Med., 32 (2003), pp. 513-521.

Lawall \& Crivelini, 2006. M.A. Lawall, M.M. Crivelini. PCNA and p53 expression in oral leukoplakia with different degrees of keratinisation. J. Appl. Oral Sci., 14 (2006), pp. 276-280.

Lippman et al., 2005. S.M. Lippman, J. Sudbo, W.K. Hong. Oral cancer prevention and the evolution of moleculartargeted drug development. J. Clin. Oncol., 23 (2005), pp. 346-356. 
Livak \& Schmittgen, 2001. K.J. Livak, T.D. Schmittgen. Analysis of relative gene expression data using Real-Time quantitative PCR and the $2^{-\Delta \Delta \mathrm{Ct}}$ method. Methods, 25 (2001), pp. 402-408.

López-Martínez et al., 2002. M. López-Martínez, M. Anzola, N. Cuevas, et al. Aplicaciones clínicas del diagnóstico de las alteraciones de p53 en el carcinoma escamoso de cabeza y cuello (p53 en el CECC). Med. Oral, 7 (2002), pp. 108-120.

Mestre et al., 1999. J.R. Mestre, G. Chan, F. Zhang, et al.. Inhibition of cyclooxygenase-2expression An approach to preventing head and neck cancer. Ann N Y Acad Sci, 889 (1999), pp. 62-71.

Mohan \& Epstein, 2003. S. Mohan, J.B. Epstein. Carcinogenesis and cyclooxygenase: the potential role of COX-2 inhibition in upper aerodigestive tract cancer. Oral Oncol., 39 (2003), pp. 537-546.

Moraitis et al., 2005. D. Moraitis, B. Du, M.S. de Lorenzo, et al.. Levels of Cyclooxygenase-2 are increased in the oral mucosa of smokers: evidence for the role of epidermal growth factor receptor and its ligands. Cancer Res., 65 (2005), pp. 664-670.

Nishimura et al., 2000. G. Nishimura, S. Yanoma, K. Satake, et al. An experimental model of tumor dormancy therapy for advanced head and neck carcinoma. Jpn J. Cancer Res., 91 (2000), pp. 1199-1203.

Pandey et al., 2008. M. Pandey, O. Prakash, W.S. Santhi, et al.. Overexpression of COX-2 gene in oral cancer is independent of stage of disease and degree of differentiation. Int. J. Oral Maxillofac. Surg., 37 (2008), pp. 379383.

Raymond et al., 2000. E. Raymond, S. Faivre, J.P. Armand. Epidermal growth factor receptor tyrosine kinase as a target for anticancer therapy. Drugs, 60 (2000), pp. 15-23.

Renkonen et al., 2002. J. Renkonen, H. Wolff, T. Paavonen. Expression of cyclo-oxygenase-2 in human tongue carcinoma and its precursor lesions. Virchows Arch., 440 (2002), pp. 594-597.

Rhee et al., 2004. J.C. Rhee, F.R. Khuri, D.M. Shin. Advances in chemoprevention of head and neck cancer. Oncologist, 9 (2004), pp. 302-311.

Roche, 2005. Roche Applied Science [database on the Internet] [cited December 20, 2005]. Assay Design Center/ProbeFinder. Homo sapiens (Human). Available from http://www.universalprobelibrary.com

Roche Applied Science, 2005. Roche Applied Science [database on the Internet] [cited December 20, 2005]. Universal ProbeLibrary. Universal ProbeLibrary interest site. Assay Design Center/ProbeFInder. Homo sapiens (Human). Available from http://www.roche-applied-science.com

Sakamoto et al., 2004. T. Sakamoto, K. Kondo, T. Yamasoba, et al. Elevated expression of cyclooxygenase-2 in adenocarcinoma of the parotid gland: insights into malignant transformation of pleomorphic adenoma. Ann. Otol. Rhinol. Laryngol., 113 (2004), pp. 930-935.

Sambrook et al., 1989. J. Sambrook, T. Maniatis, E.F. Fritsch. Molecular Cloning: A Laboratory Manual. Cold Spring Harber Laboratory Press, New York (1989).

Santhi et al., 2006. W.S. Santhi, P. Sebastian, B.T. Varghese, et al. NF-kB and COX-2 during oral tumorigenesis and in assessment of minimal residual disease in surgical margins. Exp. Mol. Pathol., 81 (2006), pp. 123-130.

Santos Garcia et al., 2005. A. Santos Garcia, M.M. Abad-Hernández, E. Fonseca-Sánchez, et al. Proteic expression of p53 and cellular proliferation in oral leukoplakias. Med. Oral Patol. Oral Cir. Bucal, 10 (2005), pp. 1-8.

Sheng et al., 1998. H. Sheng, J. Shao, J.D. Morroww, et al. Modulation of apoptosis and Bcl/2 expression by prostaglandin $\mathrm{E}_{2}$ in human colon cancer cells. Cancer Res., 8 (1998), pp. 362-366.

Shin et al., 1994. D.M. Shin, J.Y. Ro, W.K. Hong, et al.. Dysregulation of epidermal growth factor receptor expression in premalignant lesions during head and neck tumorigenesis. Cancer Res., 54 (1994), pp. 3153-3159.

Shirasuna et al., 1991. K. Shirasuna, Y. Hayaskido, M. Sugiyama, et al. Immunohistochemical localization of epidermal growth factor (EGF) and EGF receptor in human oral mucosa and its malignancy. Virchows Arch. A Pathol. Anat. Histopathol., 418 (1991), pp. 349-353.

Slaughter et al., 1953. D.L. Slaughter, H.W. Southwick, W. Smejal. Field cancerization in oral stratified squamous epithelium; clinical implications of multicentric origin. Cancer, 6 (1953), pp. 963-968.

Srinivasan \& Jewell, 2001. M. Srinivasan, S.D. Jewell. Evaluation of TGF-alpha and EGFR expression in oral leukoplakia and oral submucous fibrosis by quantitative immunohistochemistry. Oncology, 61 (2001), pp. 284292.

Subbaranmaiah et al., 1999. K. Subbaranmaiah, I. Norton, W. Gerald, et al. Increased expression of cyclooxygenases2 in HER-2/neu overexpressing human breast cancer cells. National Cancer Institute $7^{\text {th }}$ Spore Investigators Workshop, Rockville, MD (1999).

Sudbo \& Reith, 2005. J. Sudbo, A. Reith. The evolution of predictive oncology and molecular-based therapy for oral cancer prevention. Int. J. Cancer, 115 (2005), pp. 339-345.

Taketo, 1998. M.M. Taketo. Cyclooxygenase-2 inhibitors in tumorigenesis. J. Natl Cancer Inst., 90 (1998), pp. 16091620.

Terakado et al., 2004. N. Terakado, S. Shintani, J. Yano, et al. Overexpression of cyclooxygenase-2 is associated with radioresistance in oral squamous cell carcinoma. Oral Oncol., 40 (2004), pp. 383-389.

Tsai et al., 2004. W.C. Tsai, S.T. Tsai, J.Y. Ko, et al. The mRNA profile of genes in betel quid chewing oral cancer patients. Oral Oncol., 40 (2004), pp. 418-426.

Tsujii \& DuBois, 1995. M. Tsujii, R.N. DuBois. Alterations in cellular adhesion and apoptosis in epithelial cells overexpressing prostaglandin endoperoxide synthase-2. Cell, 83 (1995), pp. 493-501.

Turini \& Dubois, 2002. M.E. Turini, R.N. Dubois. Cyclooxygenase-2: a therapeutic target. Ann Mev. Med., 53 (2002), pp. 35-57.

Vadlamudi et al., 1999. R. Vadlamudi, M. Mandal, I. Adam, et al. Regulation of cyclooxygenase-2 pathway by HER2 receptor. Oncogene, 18 (1999), pp. 305-314. 
Vora et al., 2006. H.H. Vora, T.I. Trivedi, S.N. Shukla, et al. p53 expression in leukoplakia and carcinoma of the tongue. Int. J. Biol. Markers, 21 (2006), pp. 74-80.

Wiese et al., 2001. F.W. Wiese, P.A. Thompson, F.F. Kadlubar. Carcinogen substrate specificity of human COX-1 and COX-2. Carcinogenesis, 21 (2001), pp. 5-10.

Williams et al., 2000. C.S. Williams, M. Tsujii, J. Reese, et al. Host cyclooxygenase-2 modulates carcinoma growth. J. Clin. Invest., 105 (2000), p. 1598-1594.

Xie \& Herchman, 1995. W. Xie, H.R. Herchman. v-src induces prostaglandin synthase 2 gene expression by activation of the c-Jun N-terminal kinase and the c-Jun transcription factor. J. Biol. Chem., 270 (1995), pp. $27622-27628$.

Ye et al., 2004. F. Ye, J. Wu, T. Dunn, et al. Inhibition of cyclooxygenase-2 activity in head and neck cancer cells by genistein. Cancer Lett., 211 (2004), pp. 39-46. 